\title{
2,5-Bis(p-R-arylethynyl)rhodacyclopentadienes Show Intense Fluorescence: Denying the Presence of a Heavy Atom**
}

\author{
Andreas Steffen, Meng Guan Tay, Andrei S. Batsanov, Judith A. K. Howard, Andrew Beeby,* \\ Khuong Q. Vuong, Xue-Zhong Sun, Michael W. George,* and Todd B. Marder*
}

The photophysics and photochemistry of transition-metal compounds are of great interest, particularly since such materials have been exploited for a wide range of applications including photocatalysis, photosynthesis and photosynthetic model compounds, artificial light-harvesting antenna systems for solar energy conversion, sensing and imaging, supramolecular photochemically driven machines, multiphotonabsorption materials, probes for monitoring biological processes, and the fabrication of high-performance organic lightemitting diodes (OLEDs). ${ }^{[1]}$ A full understanding of the excited-state behavior of organometallic compounds is crucial for the design of new materials for all of these applications. An attractive feature of this class of compounds is that subtle changes in the ligand environment or metal can be used to tune the properties, thereby allowing the control required for a particular application. Diimine complexes of $\mathrm{Ru}^{\mathrm{II}}, \mathrm{Re}^{\mathrm{I}}$, and $\mathrm{Pt}^{\mathrm{II}}$ have been extensively studied. ${ }^{[2]}$ Recently there has also been considerable interest in the photophysics of $\mathrm{C}^{\wedge} \mathrm{N}$ cyclometalated complexes, particularly $\operatorname{Ir}^{\mathrm{III}},{ }^{[1,3]}$ and both the diimine and $\mathrm{C}^{\wedge} \mathrm{N}$ cyclometalated complexes can exhibit highly emissive triplet excited states.

Mononuclear metal complexes usually show very rapid conversion from singlet into triplet excited states, which is attributed to the "heavy-atom effect". The heavy-atom effect is the promotion of intersystem crossing (ISC) processes by the spin-orbit coupling (SOC) of the metal atom. These effects can begin to be observed with elements as light as sulphur $(z=16) \cdot{ }^{[4]}$ For example, the formation of the ${ }^{3}$ MLCT (MLCT $=$ metal-to-ligand charge transfer) excited state of $\left[\mathrm{Ru}(\mathrm{bpy})_{3}\right]^{2+}\left(\mathrm{bpy}=2,2^{\prime}\right.$-bipyridine $)$ occurs on a timescale of

[*] Dr. A. Steffen, M. G. Tay, Dr. A. S. Batsanov, Prof. Dr. J. A. K. Howard, Dr. A. Beeby, Prof. Dr. T. B. Marder

Department of Chemistry, Durham University

South Road, Durham, DH1 3LE (UK)

Fax: (+44) 191-384-4737

E-mail: andrew.beeby@durham.ac.uk todd.marder@durham.ac.uk

Homepage: http://www.dur.ac.uk/chemistry/staff/profile/?id = 194

Dr. K. Q. Vuong, Dr. X.-Z. Sun, Prof. Dr. M. W. George

School of Chemistry, University of Nottingham

University Park, Nottingham, NG7 2RD (UK)

Fax: (+ 44) 115-951-3555

E-mail: mike.george@nottingham.ac.uk

[**; A.S. thanks the DAAD and the EU (Marie-Curie) for postdoctoral fellowships. M.W.G. gratefully acknowledges receipt of a Wolfson Merit Award. M.G.T. thanks the Universiti Malaysia Sarawak for a Ph.D. scholarship.

Ð) orting information for this article is available on the WWWW under http://dx.doi.org/10.1002/anie.200905697. less than $20 \mathrm{fs}^{[5]}$ The precise factors governing the singlet-totriplet excited state interconversion have recently been questioned by observations which have shown that formation of the ${ }^{3} \mathrm{MLCT}$ state of the first-row complex $\left[\mathrm{Fe}(\mathrm{bpy})_{3}\right]^{2+}$ occurs in less than $20 \mathrm{fs}$, whereas the second-row complexes $\left[\operatorname{Re}(\mathrm{X})(\mathrm{CO})_{3}(\mathrm{bpy})\right]^{+}(\mathrm{X}=\mathrm{Cl}, \mathrm{Br}, \mathrm{I})$ show a much slower interconversion (ca. $100 \mathrm{fs}){ }^{[6]}$ Furthermore, the order was found to be $\mathrm{Cl}(85 \mathrm{fs})<\mathrm{Br}(128 \mathrm{fs})<\mathrm{I}(152 \mathrm{fs})$, which is contrary to that predicted by the simplistic consideration of the effect of the heavy atom. Tetrahedral [Pt(binap $\left.)_{2}\right]$ (binap $=2,2^{\prime}$-bis(diphenylphosphino)-1,1'-binaphthyl) and $[\mathrm{Cu}\{$ bis(diimine $)\}]^{+}$complexes have been shown to have unusually long-lived ${ }^{1}$ MLCT states of $\tau=3 \mathrm{ps}$ and $\tau=15 \mathrm{ps}$, respectively, attributed to a distortion towards a squareplanar geometry which reduces the mixing of the ${ }^{1,3}$ MLCT states. $^{[7]}$

Our long-standing interest in rhodium-acetylide compounds $^{[8]}$ and luminescent bis(arylethynyl)arenes ${ }^{[9]}$ led us to the development of a high-yielding, one-pot synthesis of a 2,5bis(phenylethynyl)rhodacyclopentadiene, which we reported to be luminescent. ${ }^{[10]}$ Our subsequent investigations, reported herein, indicate that this new class of luminescent rhodium complexes shows unprecedented excited-state behavior. Our luminescence spectroscopic studies are supported by picosecond time-resolved IR (TRIR) vibrational spectra of the ground and excited states as a means by which to obtain accurate kinetic data on the processes involved. Herein we demonstrate that despite the presence of the second-row transition metal the compounds show remarkable photophysical properties: specifically, long-lived, highly emissive singlet excited states. This new class of material challenges our understanding of the behavior of excited electronic states and the role of the heavy atom in intersystem-crossing processes.

The reaction of $\left[\mathrm{Rh}\left(\mathrm{C} \equiv \mathrm{CSiMe}_{3}\right)\left(\mathrm{PMe}_{3}\right)_{3}\right]$ (1) with the bis(diyne)s $\mathbf{2 a - d}$ leads to the formation of the metallacyclic complexes 3a-d (Scheme 1, top), which have been unambiguously characterized by ${ }^{1} \mathrm{H}$ and ${ }^{31} \mathrm{P}$ NMR and IR spectroscopy, mass spectrometry, elemental analysis, and by singlecrystal X-ray diffraction studies on 3a-c (Figure 1). In situ NMR spectroscopic studies show that the reactions occur quantitatively, and the products have been isolated in 23$82 \%$ yield after several recrystallizations, to ensure high purity for photophysical studies. The photophysical data are summarized in Table 1 and Table 2.

Compounds $\mathbf{3 a}-\mathbf{d}$ absorb light with extinction coefficients of $15000-44000 \mathrm{~L} \mathrm{~mol}^{-1} \mathrm{~cm}^{-1}$ and emit in the visible region (Figure 2). A vibrational progression typical of aromatic stretching modes (ca. $1360 \mathrm{~cm}^{-1}$ ) is observable in the absorp- 